Revista General de Información y Documentación ISSN: 1132-1873

https://dx.doi.org/10.5209/rgid.66970

\title{
Las Epistolae Magni Turci de Laudivio Zacchia en dos ediciones incunables españolas: nuevos datos para su identificación ${ }^{1}$
}

\author{
Arantxa Domingo Malvadi ${ }^{2}$
}

Recibido: 11 de diciembre de 2019 / Aceptado 24 de marzo de 2020

Resumen. Las Epistolae Magni Turci de Laudivio Zacchia, durante mucho tiempo atribuidas a Mehmet II, tuvieron un notable éxito editorial en el siglo XV de forma que se llebgaron a imprimir hasta en 20 ocasiones entre 1473 y 1500. En España se conocían hasta el momento 2 ediciones: la primera, sin datos de identificación editorial pero atribuida al taller de Pablo Hurus en Zaragoza en torno a 1480-1484, y otra edición fechada en 1490 y adscrita al taller de Heinrich Botel en Lérida. En las líneas que se siguen se revela la existencia de una nueva edición de la obra que no figura en los repertorios bibliográficos, cuya identificación y adscripción al taller de Pablo Hurus, obliga a una reinterpretación de los datos existentes sobre las ediciones incunables de la obra en España y los ejemplares conservados.

Palabras clave: Zacchia; Mehmet II; imprenta incunable; incunables españoles; historia de la imprenta; historia del libro; Pablo Hurus; Zaragoza; Barcelona; Nápoles; Rodas; Mediterráneo.

\section{[en] Laudivius Zacchia’s Epistolae Magni Turci in two Spanish Early Printed Books: New data for identification}

\begin{abstract}
Laudivio Zacchia’s Epistolae Magni Turci, for a long time attributed to Mehmet II, were destined to become a bestseller in the Early printing Europe with about twenty editions between 1473 and 1500. Two Spanish editions were known until now: one, unsigned, was attributed to Paul Hurus' workshop in Zaragoza circa 1480-1484; the other, dated in 1490, to Botel's workshop in Lérida. A third unknown edition of the work, not mentioned in the bibliographical repertoires, is now identified and attributed also to Hurus' workshop. A new assessment is made of the available information on the Spanish first editions of this work and their preserved copies.
\end{abstract}

Keywords: Zacchia; Mehmed II; early printed books; Spanish incunabula; printing history; book history; Paul Hurus; Zaragoza; Barcelona; Naples; Rhodes; Mediterranean.

Sumario. 1. Introducción. 2. Estado de la Cuestión. 3. Una nueva edición de las Epistolae Magni Turci. 4, Adscripción de las ediciones al taller de Hurus. 5. Conclusiones. 6. Referencias bibliográficas.

1 Este artículo forma parte de los resultados del Proyecto de I+D del Ministerio de Economía y Competitividad FFI2016-78245-P “Repertorio de Incunables Españoles”.

2 Real Biblioteca

E-mail: arantxa.domingo@patrimonionacional.es 
Cómo citar: Domingo Malvadi, A. (2020) Las Epistolae Magni Turci de Laudivio Zacchia en dos ediciones incunables españolas: nuevos datos para su identificación, en Revista General de Información y Documentación 30 (1), 129-148.

\section{Introducción}

A finales del siglo XV vieron la luz sucesivas ediciones de las Epistolae Magni Turci, una colección de cartas que supuestamente había escrito Mehmet II a distintos príncipes y pueblos del Mediterráneo, acompañadas de las respuestas de estos; se presentaban traducidas por Laudivius Zacchia al latín del sirio, del griego y del escita, lenguas en las que estarían escritas originalmente: "epistolas vero ad innumeras orbis gentes plurimas dictavit, parti Syro et Greco sermone compositas, partim etiam Scythica lingua scriptas. Quas quidem Laudivius eques Hierosolimitanus earum post sententias assecutus latinas edidit”. Aunque las cartas no estaban datadas era claro que se habrían escrito entre la conquista de Constantinopla en 1453 y la toma de Negroponte en 1471, que no aparece mencionada.

Aunque en el último cuarto del siglo XIX ya se consideraba autor de las cartas a Laudivio Zacchia da Vezzano (Neri, 1875: 137-153), su autoría quedó totalmente demostrada en el siglo pasado (Babinger, 1960: 1-43) y en la actualidad ya nadie duda de que la atribución de las cartas al propio Mehmet II no fue sino una falsificación literaria similar a la de las cartas en griego del tirano Falaris (s. VI a.C.) esclarecida en el siglo XVII (Bentley, 1697). La edición conjunta de ambas obras (Deventer: Richardus Pafraet, 1491-1492) vendría a confirmar el carácter literario y retórico de ambas colecciones (ISTC: im00063600). En el mismo periodo se publicaron una serie de obras conectadas con la presencia turca en el Mediterráneo tras 1453, la toma de Negroponte (1471), el asedio de Rodas (1480) y la toma de Otranto (1480).

La primera edición de la obra vio la luz en Nápoles el 17 de septiembre de 1473 en el taller de Arnaldo de Bruxelles, activo en dicha ciudad entre 1472 y 1477 (Tentori, 1962). La obra se volvió a editar el 27 de noviembre 1473 en Roma por Giovanni Filippo da Lignamine (Alaimo, 1988) y hasta diecisiete veces más en el último cuarto del siglo. En el contexto histórico en que se produjo la obra, tras la toma de Constantinopla y con el turco tomando posiciones en el Mediterráneo, se entiende que se convirtiera en un auténtico best-seller que circuló tanto en copias manuscritas como impresas con traducciones posteriores a otras lenguas (Meserve, 2008: 227-229).

Entre la veintena de ediciones del siglo XV consignadas hasta el momento en los repertorios bibliográficos figuran dos ediciones españolas, una fechada en 1490 y adscrita al taller de Heinrich Botel en Lérida (ISTC: im00063500) y otra anterior, adscrita al taller zaragozano de Hurus, entre 1481-1484, objeto de nuestro interés (ISTC: im00059500). 


\section{Estado de la Cuestión}

La noticia de la existencia de un ejemplar de la primera edición hispana conservado en la BNE aparecía en el primer catálogo de incunables de la institución (García Rojo, 1945: 288, n 1125). La descripción bibliográfica remite a un ejemplar sin datos bibliográficos. Poco después, Vindel en su Arte tipográfico en España recogía la noticia de esta, hasta aquel momento, desconocida edición de las Epistolae Magni Turci de Laudivius Zacchia representada en el ejemplar de la BNE y la adscribía a la imprenta zaragozana de Pablo Hurus y Johannes Planck, entre 1480-1484 (Vindel, 1946: 23-24, nº 6). Esta es la descripción bibliográfica que ofrece:

Pablo Hurus y Juan Blanco (Planck) (Primera época): Laudivius. Epistolae Magni Turci Mahomet II. Sin indicaciones tipográficas pero Zaragoza, Pablo Hurus y Juan Blanco (Planck) hacia 1479-1484).

Sign. A1: Laudivii equitis hierosolymitani ad / Francinū Beltrandū in epistolas magni / Turci praefatio. Signaturas: a-b8, c6, d8. Letra romana. Tipografía de un solo tamaño. 21-23 líneas por plana. Huecos en blanco para las capitales.

Desconocido a Haebler.

Ejemplar en la BN de Madrid, signatura I/349. En el Catálogo de incunables de la BNE, bajo el núm. 1125 se describe por primera vez este desconocido libro y que nosotros identificamos como impreso con la tipografia de Pablo Hurus empleada en Barcelona en 1475 y más tarde en Zaragoza en la foliación de los Fueros, $\mathrm{n}^{\circ} 3$, y del Vita et Transitus $\mathrm{n}^{\circ} 4$ y en los textos de Casus Papales $\mathrm{n}^{\circ}$ 8, de la Bula $\mathrm{n}^{\circ} 9$ y del Caoursin, $\mathrm{n}^{\circ} 10$.

Vindel (1945: 15-24, no 1-4) se refiere a la tipografía romana R110, utilizada por Johannes de Salzburgo y Pablo Hurus en dos ediciones fechadas en 1475 en el taller que ambos tuvieron en Barcelona, la de los Opera de Salustius (ISTC: is00060300) y el Epitome de Florus (ISTC: if00233500), así como en la edición sin data de la obra de Cicerón In Catilinam Orationes (ISTC: ic00551950). La misma tipografía R110 aparece en los Rudimenta grammaticae de Nicolaus Perottus (ISTC: ip00304800), esta sí una edición con datos en el colofón que la sitúan con claridad en Barcelona, en el taller de Juan de Salzburgo y Pablo de Constanza el 12 de diciembre de 1475: "in Barcynonis...a Johanne de Salsburga et Paulo de Constantia Germanis qui tum ibi forte aderant imprimi curarunt...quod fuit perfectum pridie idus Decembris Anno Christiane salutis M.CCCC.LXXV. Deo gratias".

Esta tipografía romana R110 es la utilizada en la primera edición española de las Epistolae Magni Turci de Laudivius Zacchia (Véase Figura 1). Pero al carecer de fecha Vindel la sitúa no en Barcelona en 1475, sino en Zaragoza donde Hurus vuelve a utilizar esta tipografía R110 en la foliación de Fori Regni Aragonum y Vita et Transitus y en el texto de varias obras que salen de su taller entre 14791482. Una de estas ediciones, datada en 1481, es la obra de Guillermo Caorsin, Obsidionis Rhodiae urbis descriptio, que narra el asedio a la isla de Rodas en 1480 y que por su tema justificaría la edición de las Epistolae Magni Turci en Zaragoza. Las otras ediciones están sin data, pero el uso de la tipografía romana R110 ha 
llevado a adscribirlas al taller de Hurus en Zaragoza entre 1480-1484: Casus Papales et Episcopales (ISTC: ic00259300), el Tractatus de ieiuniis de Antonio Rosellis (ISTC: ir00323300), la Bula de indulgencias a favor de la Orden de Rodas o la Epistola ad Rabbi Isaac de prophetiis Veteris Testamenti contra errores Iudaeorum de Samuel Marochitanus (ISTC: is00108500) que no menciona Vindel.

El Catálogo general de incunables en Bibliotecas españolas (IBE, 1989: 550, $\mathrm{n}^{\circ}$ 3457) señala un único ejemplar en la BNE y, siguiendo a Vindel, lo adscribe a Zaragoza entre 1480-1484.

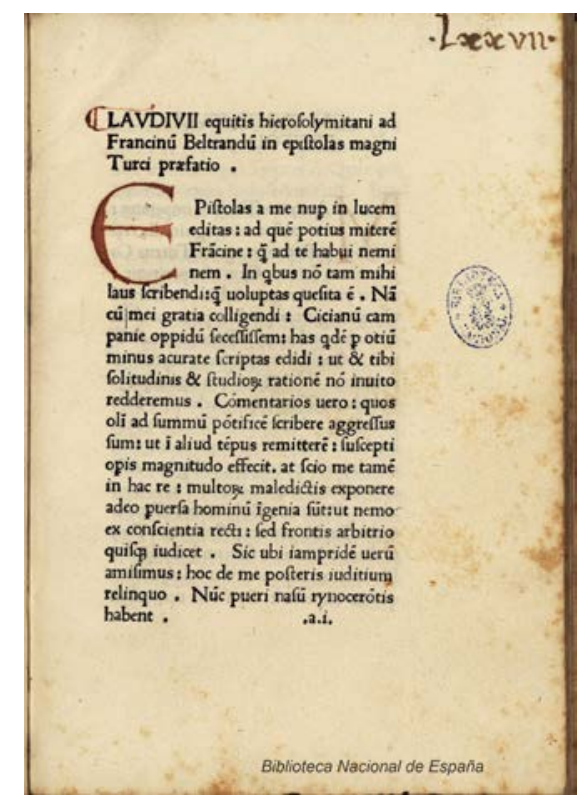

Figura 1. Epistolae Magni Turci. [s.l.: s.d., s.a.]

Ejemplar BNE I/2588. Letra romana R110

Por su parte, el Gesamtkatalog der Wiegendrucke (GW: M25638), frente a la opinión de Vindel y, siguiendo probablemente a Griffin (1988: 17) en su análisis de los incunables españoles existentes en la biblioteca John Rylands, considera la edición de las Epistolae Magni Turci como impresa en Barcelona en 1475. Y menciona un total de cuatro ejemplares de la edición: el conservado en la John Rylands Library de Manchester, dos ejemplares conservados en la BNE (uno de ellos el mencionado por Vindel) y el cuarto conservado en la Biblioteca Vaticana.

El escollo que supone determinar el lugar y la fecha de edición está lejos de estar cerrado. Así lo refleja la nota que figura en el ISTC para esta edición incunable (ISTC: im00059500): "The types are identified as those used at Zaragoza by Vindel (followed by BMC X p.26 and IBE), but as those used at Barcelona at JRL (N. Griffin, in Bulletin of the John Rylands University Library, 70:2 (1988), p.17)”. En efecto, Griffin, en el mencionado estudio, se apoya en la similitud de la edición con la de los Rudimenta Grammatices de Nicolao Perotti y señala que las Epistolae Magni Turci fueron impresas "In the same roman type found in the 
Nicolaus Perottus (or Perothus) Rudimenta grammaticae of 12 December 1475 (Haebler 542(8); IB52505; Vindel I. 21-2, $n^{0}$ 3; unique copy in Zarazoza BCat), for long believed to be the first book printed at Barcelona, and described in its colophon (sig. r10a) as having been printed by Ioannes de Salsburga and Paulo de Constantia qui ibi forte aderant"y añade "The same roman type used in both was also employed in three unsigned editions: a Florus Epitome... a Sallust, both of 1475 and an undated Cicero, In Catilinam invectivae; some of it reappears in the Caoursin, Rodiae obsidionis descriptio of 28 February/1 March 1481”.

Al tratarse del mismo tipo, Griffin parece utilizar los mismos argumentos que emplea Vindel para situar la edición en Zaragoza, ciudad a la que se trasladó Hurus a finales de 1475 o principios de 1476 (Pallarés, 2008: 61-62). Si se opta por Barcelona hay que situar el impreso antes de la marcha de Hurus a Zaragoza a finales de 1475. Si se opta por Zaragoza, la impresión hay que situarla entre 1479 y 1482, cuando del taller de Hurus salen obras impresas con la tipografía romana R110. Volveremos sobre el tema más adelante. Conviene terminar de exponer los datos que ofrece la bibliografía de referencia.

Al igual que el $G W$ el ISTC señala para esta edición los siguientes ejemplares: dos ejemplares en la BNE (Madrid) (I/359 y I/2588), uno en la Biblioteca Vaticana encuadernado con otras obras (Stamp.Chig.IV.1251, 3) y el cuarto en la John Rylands University Library de Manchester (JRL 18486).

En el apartado destinado a la bibliografía de esta edición el ISTC recoge dos referencias del $G W$ : la primera remite a esta edición (GW: M25638); la segunda ( $G W$ : M25618) hace referencia a una edición de la misma obra, las Epistolae Magni Turci, sin datos bibliográficos, pero igualmente adscrita al taller de Hurus y Planck en Zaragoza entre 1481-1484, de la que se menciona un ejemplar en la Biblioteca de Cataluña. La ficha manuscrita que describe el ejemplar es muy somera: 24 h., tipografía 103G. No se da más información, de tal manera que no queda clara su conexión con la edición de las Epistolae Magni Turci en caracteres romanos descrita en el cuerpo de la ficha.

\section{Una nueva edición de las Epistolae Magni Turci}

El Catálogo Colectivo del Patrimonio Bibliográfico Español recoge la edición de las Epistolae Magni Turci de la que venimos haciendo mención (CCPB: 000110692-9). Siguiendo a Vindel y al IBE, la adscribe al taller de Hurus y Planck en Zaragoza, entre 1480-1484. Entre los ejemplares de la edición conservados en España recoge uno de los dos ejemplares conservados en la BNE, el I/359, y omite el I/2588. Incluye, en cambio, el conservado en Barcelona, en la Biblioteca de Cataluña (9-V-62). Del CCPB parece desprenderse que estamos ante un nuevo ejemplar de la edición de las Epistolae Magni Turci que podemos añadir a la lista de ejemplares conservados en las bibliotecas extranjeras.

La digitalización del ejemplar a través de Memòria Digital de Catalunya permite comprobar que se trata de una edición diferente (Véase Figura 2) y que, al igual que en el caso anterior, estamos ante un ejemplar huérfano de datos 
tipográficos, por lo que su asignación al taller debe hacerse tras un análisis de su tipografía.

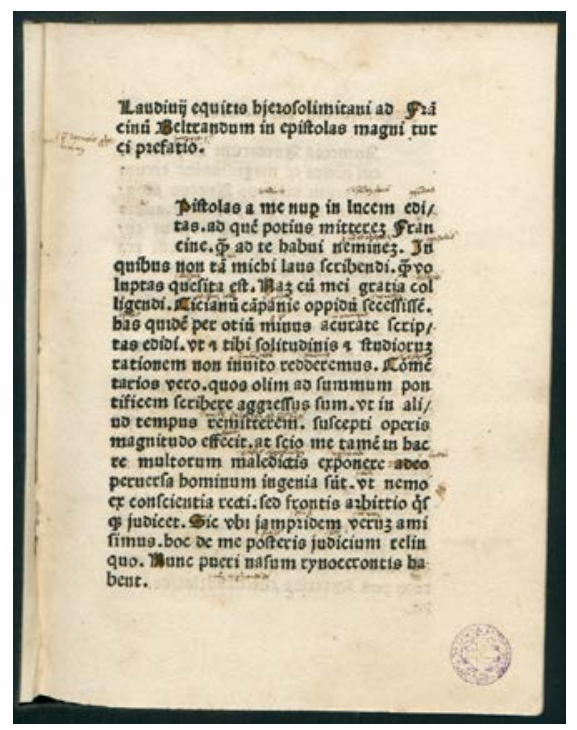

Figura 2. Epistolae Magni Turci. [s.l.: s.d., s.a.]

Ejemplar BC, 9-V-62. Letra Gótica G104

Se trata de una edición en cuarto, que ocupa, no 30 h., sino 24 h., sin numerar. Al contrario que la edición anterior, no presenta signaturas, aunque la colación más factible parece $\left[a-c^{8}\right]$. La tipografía utilizada es una letra gótica, que el $T W$ señala como del tipo 1: 104G: M17E, tipografía gótica usada en Zaragoza por el taller de Turrecremata en el que colaboraban Enrique Botel, Pablo Hurus y Juan Planck.

El taller utilizará en distintos momentos hasta tres tipografías distintas asociadas a la 104G. Las tres se diferencian por algunos detalles claros, especialmente visibles en las mayúsculas que aparecen recogidos en el TW:

a. Tipo 1*104G, M17E usado en 1477, probablemente cuando Botel trabaja en el taller. Con este tipo se imprime la Monitoria para que los musulmanes de Aragón y Navarra derriben las Zomas y los Fori Regni Aragonum (Véase Figura $3)$ : 


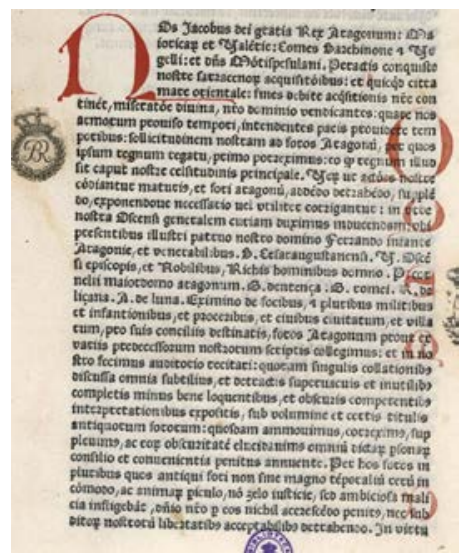

Figura 3. Fori Regni Aragonum.

[Zaragoza: Henricus Botel et Paulus Hurus, 1477], Tipo 1*104G, M17E

b.Tipo 1: 104G, M17E usado entre 1481-1483. Empleado en algunas ediciones de Hurus, algunas con data, como la Bula de indulgencias en favor de la Santa Cruzada para la defensa de Rodas (1479) en castellano y en latín, la Bula de indulgencias en favor de la Orden de la Merced, para vivos, en latín, fechada en 1481 en sus dos composiciones (ISTC: ir00202650, ISTC: ir00202600), el Psalterium latinum cum canticis, con data en Zaragoza en 1481 (ISTC: ip01042300); y otras sin data, pero situadas entre 1480-1484: el Arte de bien morir con el confesionario breve (ISTC: ia01124200), Expositio officii Missae sacrique canonis (ISTC: ie00169000), Vita Christi por coplas de Mendoza (ISTC: im00488000), la comedia Pamphilus de amore (ISTC: ip00017700) y los Elegantiarum Augustini Dati (ISTC: id00070400) (Véase Figura 4).

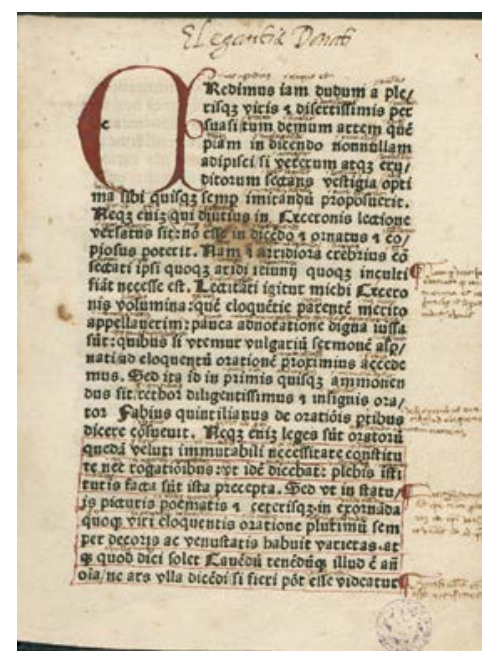

Figura 4. Augustini Dati Elegantiarum.

[Zaragoza, Hurus y Planck, c. 1481] Tipo 1 104G, M17E 
c.Tipo 2: 104G, M61, usado únicamente en la Expositio super toto psalterio, obra impresa en Zaragoza y fechada el 12 de noviembre de 1482 (ISTC: it00528000). No figura el nombre del impresor, pero se puede asignar al taller conocido como de Turrecremata en el que intervienen Enrique Botel, Pablo Hurus y Juan Planck, activo entre 1477 y 1484 (Véase Figura 5):

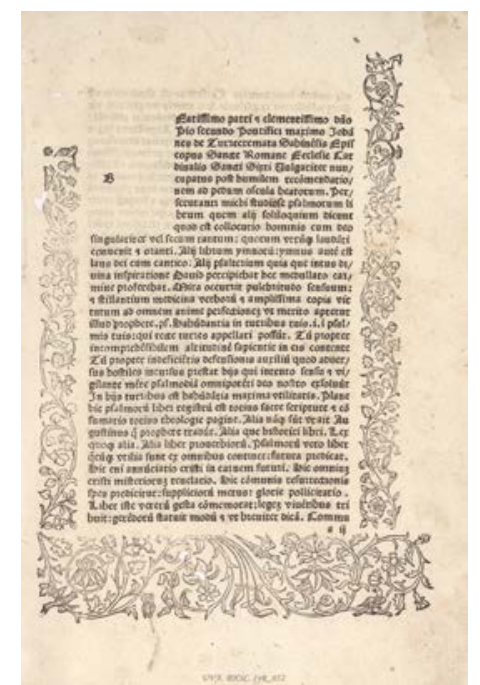

Figura 5. Johannes Turrecremata. Expositio super toto Psalterio, Zaragoza:

[Turrecremata], 1482. Tipo 2: 104G, M61

Parece claro que el tipo usado para la edición de las Epistolae Magni Turci representado en el ejemplar conservado en Barcelona, es el Tipo 1: 104G, M17E, usado también, entre otras ediciones, en las Elegantiarum de Agustino Dati (Figura 4). Se trata de un tipo bien documentado en el taller zaragozano de Hurus entre 1479 y 1484, la misma horquilla cronológica en la que se ha situado la edición de la obra en caracteres romanos.

\section{Adscripción de las ediciones al taller de Hurus}

Resulta evidente por tanto que estamos ante dos ediciones de una misma obra, ambas completas desde el punto de vista de su contenido ${ }^{3}$, ambas sin data, que la

3 Ambas comienzan con el Prefacio de Laudivio a Francesc Beltrán (Laudivii equitis hieroslymitani ad Francinum Beltrandum in epistolas magni Turci praefatio); seguido del argumento de las cartas (Argumentum Epistolarum), tras el que siguen las diferentes cartas cuyas rúbricas se transcriben a continuación: Turcus Zancassano, regi persarum; Zancassanus turco; Turcus zancassano; Zancassanus turco; Sexvares rex armenie turco; Turcus Sexvari; Soldanus turco; Turcus soldano; Turcus pontifici romano; Pontifex romanus turco; Turcus pontifici romano; Pontifex romanus turco; Turcus Venetis; Veneti turco; Turcus Grecie magistratibus; Grecie magistratus turco; Turcus venetis; Veneti turco; Turcus Genuensibus; Genuenses turco; Turcus Naritiis; Naricii Turco; Turcus corcyerensibus; Corcyrenses turco; Turcus regi cypriorum; Rex cypriorum Turco; Turcus Methonensibus; Methonenses Turco; Turcus Coronensibus; Coronenses Turco; Turcus Florentinis; Florentini Turco; Turcus Ponticis; Pontici Turco; Turcus 
bibliografia referencial o ha ignorado o ha considerado como una única edición en parte debido al sistema de descripción de los incunables y, en parte, porque en ambas ediciones se han utilizado tipografias atribuibles al taller de Hurus (Véase su descripción bibliográfica al final).

Las ediciones sucesivas de una misma obra no constituyen un caso aislado en la producción zaragozana de Hurus, al que vemos imprimir hasta dos y tres veces la misma obra: las Fabulae de Esopo (1482, 1489), el Directorium humanae vitae de Juan de Capua, traducido como Exemplario contra los engaños y peligros del mundo (1493, 1494), el Repertorio de los tiempos de Andrés de Li (1492, 1495), el Confessionale de Antoninus de Florencia (1492, 1497, c. 1499-1500) y dos ediciones del Ars moriendi en español (1481-1484, 1488-1491) y una edición en catalán (14881490).

Se trata ahora de comprobar, a partir del análisis tipográfico y del contexto cultural en el que se pudieron producir las ediciones, si realmente ambas se hicieron en la misma ciudad (Zaragoza) en el tiempo en que Hurus trabajaba con los dos tipos de letra (la R110 y la G104), es decir, entre 1479 y 1484, o en ciudades diferentes, Barcelona (R110) y Zaragoza (G104), lo que implicaría un cambio en las fechas de impresión, ya que habría que adelantar en ese caso la fecha de producción de la edición en romanos al año 1475.

En el caso de la edición en letra gótica no hay duda de su adscripción al taller zaragozano de Hurus en las fechas propuestas. Además de la tipografia, su edición en Zaragoza estaría justificada por el contexto político del momento, que coincidía con el asedio a Rodas y la publicación de las bulas para hacer frente al turco en la isla en 1481.

Es en la edición en tipos romanos utilizados por Hurus tanto en Barcelona como en Zaragoza en la que debemos poner el énfasis y centrar nuestro análisis.

A simple vista la letra romana R110 utilizada en Barcelona y la R110 utilizada en Zaragoza apenas presentan diferencias. El catálogo de incunables de la British Library (BMC, 1971: X, $\mathrm{n}^{\circ} 3$ ) señala algunos rasgos distintivos de la tipografía R110 (R111) en los impresos barceloneses de Johannes de Salsburgo y Paulo (Hurus) de Constantia: la "Q" se presenta con dos formas: una con la cola más larga que la otra; las astas de la " $M$ " ligeramente inclinadas, las astas diagonales parecen descender por debajo del nivel de la línea. La "N" parece inclinarse a la izquierda; la "h" utilizada es del tipo "Valdarfer"; el signo de interrogación es largo, con forma ligera, poco separado del punto, algo más separado en los

Cretensibus; Cretenses Turco; Turcus Chii; Chii Turco; Turcus Epidauriis; Epidauri Turco; Turcus Nauplensibus; Nauplenses turco; Turcus Pannoniis; Pannonii Turco; Turcus Macedonibus; Macedones Turco; Turcus Asiaticis; Asiatici Turco; Turcus Bithiniis; Bithinii turco; Turcus Delphicis; Delphici Turco; Turcus pergamenis; Pergameni Turco; Turcus Mitheleneis; Mithelenei Turco; Turcus Amazoniis; Amazonii Turco; Turcus Ilyricis; Ilyrici Turco; Turcus Rhodiis; Rhodii Turco; Turcus Rhodiis; Rhodii Turco; Turcus Epiris; Epiri Turco; Turcus Dalmatis; Dalmate Turco; Turcus Appulis; Appuli Turco; Turcus Brundusinis; Brundusini Turco; Turcus Ciracusanis; Syracusani Turco; Turcus Italie magistratibus; Italie Magistratus Turco; Turcus Regi Ferdinando; Rex Ferdinandus Turco; Turcus Siculis; Siculi Turco; Turcus Alexandrinis; Alexandrini Turco; Turcus Tartaris; Tartari turco; Turcus Atheniensibus; Athenienses turco; Turcus Thebanis; Thebani turco; Turcus Lacedemoniis; Lacedemonii Turco. Finis. 
Rudimenta Grammatices de Perotti; y el signo de abreviatura latina de la desinencia -us "9" con la cola debajo de la línea.

El TW señala que los rasgos más definitorios de la R110 barcelonesa son el tipo "9" de la abreviatura de -us, con la cola debajo de la línea, y el signo de interrogación sobre el punto. Estos rasgos aparecen en el Epítome de Floro (ISTC: if00233500), las obras de Salustio (ISTC: is00060300) y Cicerón mencionadas (ISTC: ic00551950) y en los Rudimenta Grammatices (ISTC: ip00304800) de Perotti ${ }^{4}$.

Se puede añadir además que en estas ediciones las rúbricas presentan letrería mayúscula, apreciándose también gran espaciado antes y después del signo de puntuación, especialmente para el punto (Véase Figura 6 y Figura 7).

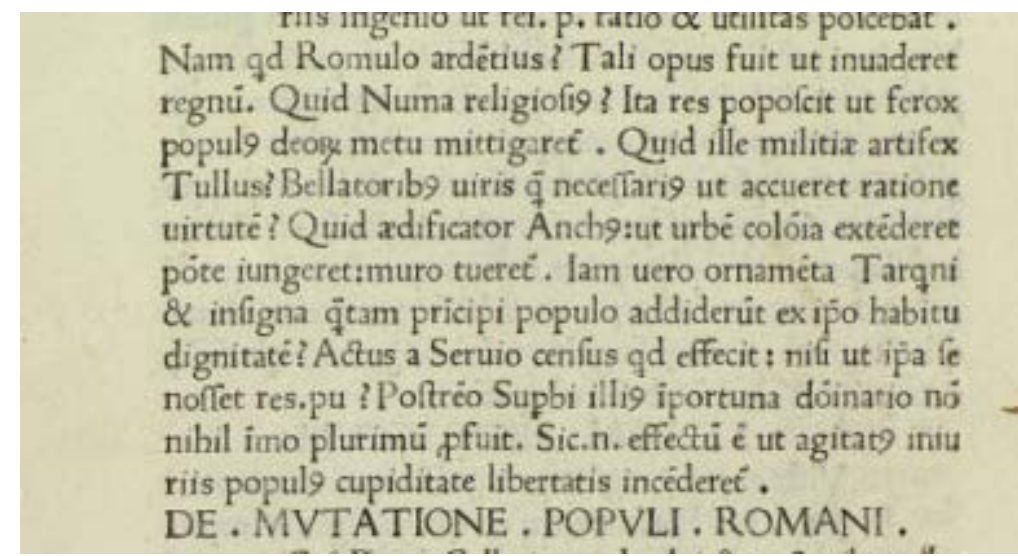

Figura 6. Florus. Epitome.

[Barcelona: Johannes Salsburga \& Paulus de Constantia], 1475.

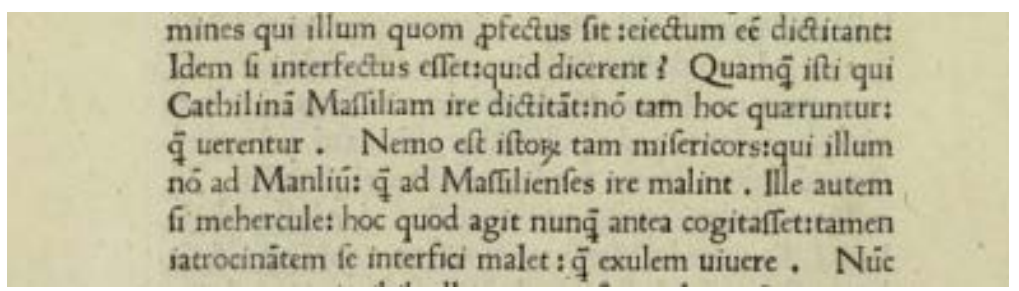

Figura 7. Cicerón. In Catilinam orationes.

[Barcelona: Johannes Salsburga \& Paulus de Constantia, 1475]

Las únicas diferencias que señala el TW en la R110 que utiliza Hurus en Zaragoza con respecto a la barcelonesa están en el signo de interrogación, que aquí es pequeño y cae directamente sobre el punto, y el uso del tipo "9" como abreviatura para la

4 Niccolò Perotti era natural de Sasoferrato. Estudió en Ferrara con Guarino de Verona. Fue secretario de Bessarion en 1447. En 1455 lo fue del papa Calixto III. En 1456 se ordena sacerdote y muy pronto es nombrado arzobispo de Siponte. Viajó por Nápoles y Alemania en misiones diplomáticas. Una biografía más amplia en Alessandro (2015). 
desinencia -us, más alto que en la tipografía R110 utilizada en el taller de Hurus en Barcelona.

Así aparece en la edición zaragozana de la obra de Caorsin, Obsidionis Rhodiae urbis descriptio (ISTC: ic00109500) fechada en 1481, en la edición de la obra de Antonio de Rosellis, De ieiuniis (ISTC: ir00323300), en el Casus papeles et episcopales (ISTC: ic00259300) o en el Tractatellus de Séneca (ISTC: is00385200), adscritas al mismo taller zaragozano entre 1480 y 1484. Se puede añadir además que frente a las ediciones barcelonesas en las que las rúbricas aparecen con letrería mayúscula, en las ediciones zaragozanas se usan tipos en minúscula en las rúbricas; por último, el tracking o espaciado entre letras tras punto es menor que en las ediciones barcelonesas (Véase Figura 8, Figura 9, Figura 10, Figura 11).

\section{funt etiä certi cafus de quibus ippi cognofcere non prit:imo de talib? criminib 9 oport3 eos neceffario ŕmittere fuos cöfitétes ad fupiorë prelatú.i..ep̃z et ifti cafus dicüé ểales:et füt ifti q feqüte quos no. Ofti.i fumma de pen.et remif. S. iiiii,verficlo, cui cófitédû. Notat eos latius SpecuP.in.iiii.pte cir ca fi.de pe.et re.5.vi.verficlo, pritata aut.}

Figura 8. Casus papales et episcopales. [Zaragoza, c. 1480-1484]

non deciate fed poffumus dicere $q$, reletramur ab iftis ppter on 9 aliogy ieiuniopy vel illopy . iiii.di erú g licet preftent ppter aliud tí nos onerant $\&$ fic in alıo releuari debem 9 . Vidédú eft modo de ieiuniis \& hoc queftiöib? vii, cxplicari credo Et quoniä ois difputatio feu locutio de ieiuniis

Figura 9. De ieiuniis. [Zaragoza, c. 1480-1484] 


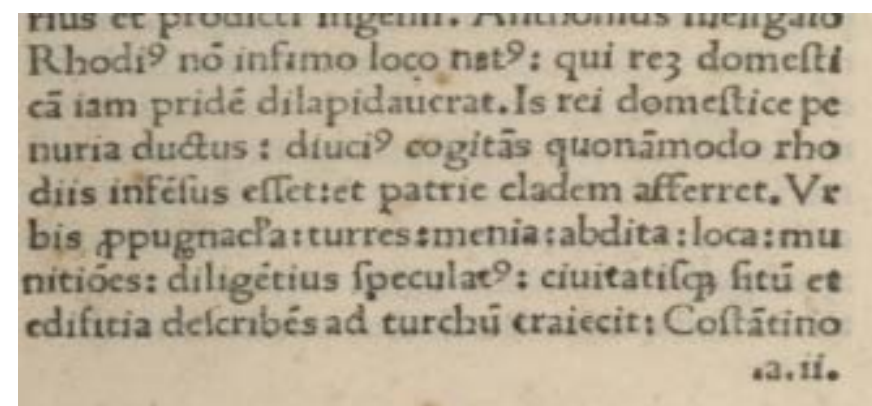

Figura 10. Caorsin. Obsidionis Rhodiae urbis Descriptio. Zaragoza, 1481.

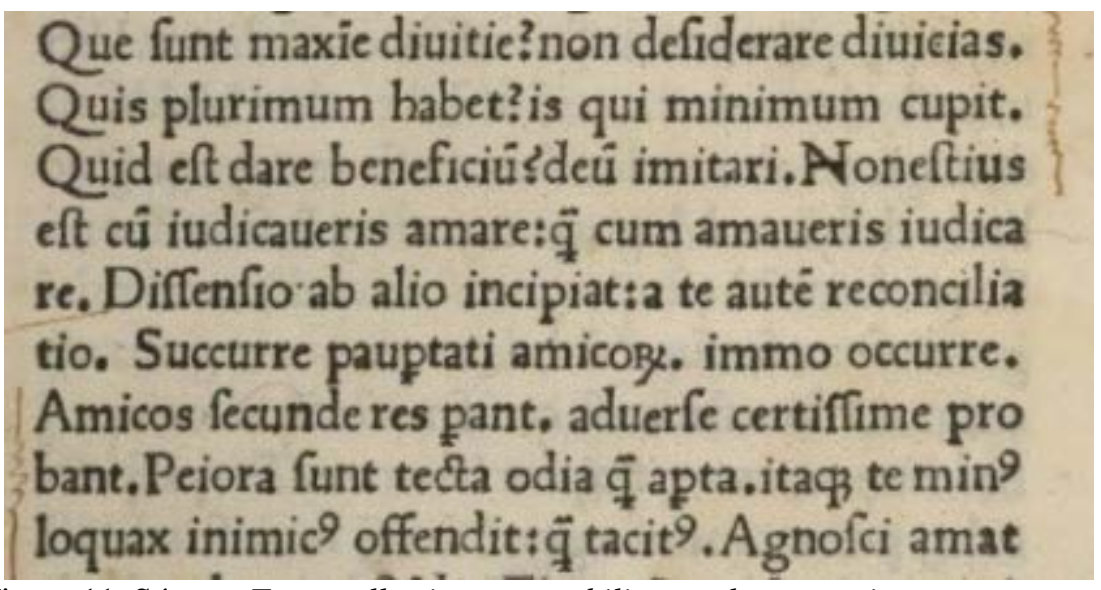

Figura 11. Séneca. Tractatellus in quo notabiliter et eleganter vite mores enarrat.

[Zaragoza, c. 1480-1484]

La comparación de la tipografía empleada en las Epistolae Magni Turci de Zacchia con la empleada en las ediciones adscritas a Barcelona y, especialmente, con la edición los Rudimenta Grammatices de Perotti, revela los mismos rasgos distintivos, especialmente evidente en el uso de letras mayúsculas en las rúbricas y el uso del tipo "9" para la abreviatura -us con la cola debajo de la línea de escritura, frente al tipo alto empleado en las ediciones zaragozanas y gran espaciado entre signo de puntuación ( . . . En cuanto al signo de interrogación, a pesar de que apenas se utiliza, en las ocasiones en que aparece, figura estilizado y separado del punto, como en los Rudimenta de Perotti (Véase Figura 12 y Figura 13). Otro rasgo coincidente es la signaturización: tanto en la edición de los Rudimenta como en la de las Epistolae las signaturas están formadas por letras y números en romanos pero en arábigos en el tercer pliego; les precede y acaban con un punto (.a.3., .b.3., .c.3., .d.3.). 


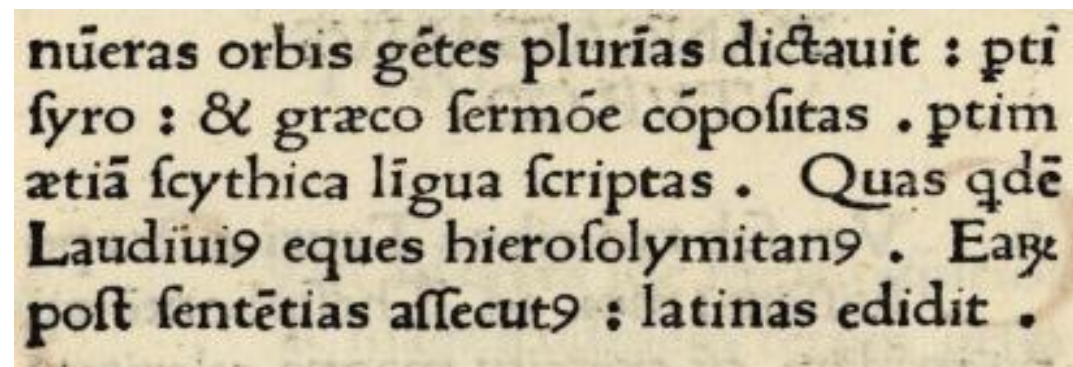

Figura 12. Laudivius Zacchia. Epistolae Magni Turci. [s.l.: s.n., s.a.]

\section{ligentif̂́me cognita nunciam9: Quis enim aduerfus eum temere quicquam au deat ? qui cum diis ipfis de imperio cer tare uideatur .}

Figura 13. Laudivius Zacchia. Epistolae Magni Turci. [s.l.: s.n., s.a.]

Estas similitudes parecen definitivas a la hora de adscribir las Epistolae Magni Turci en letra romana al taller de Hurus y Johannes de Salsburgo en Barcelona, antes de su traslado a Zaragoza, es decir, en 1475, tal y como señala Griffin.

En apoyo de esta tesis, fundamentada en el análisis de la tipografía, vendría otro argumento procedente del contexto cultural e intelectual barcelonés en el que se producen las ediciones de los Rudimenta de Perotti y de las Epistolae Magni Turci de Laudivio en 1475, que tiene como punto de contacto el reino de Nápoles durante el reinado de Alfonso V el Magnánimo y de su hijo Ferrante, quienes favorecieron el flujo comercial y cultural renacentista en la corona de Aragón a través de los súbditos aragoneses y catalanes que pasaron a Nápoles (Buffardi, 1997), entre los que podríamos situar un grupo de personas que alentarán en Barcelona la impresión de estas obras de carácter humanístico y pedagógico.

Las ediciones previas confirmadas del taller de Hurus y Johannes de Salsburgo en Barcelona en 1475 se corresponden con obras de autores clásicos latinos, Salustio, Cicerón y Floro, considerados modelos de prosa por los humanistas y profusamente utilizados en las escuelas: la Conjuración de Catilina y la Guerra de Jugurta de Salustio, los Discursos de Cicerón contra Catilina, el Epítome de Lucio Anneo Floro de la Historia de Tito Livio, y la edición de los Rudimenta Grammatices de Perotti, la primera gramática latina humanística completa, escrita en 1468, que tuvo mucho éxito en la época. Está dividida en tres partes, la definición de las partes de la oración, la morfología verbal y nominal y un tratado sobre el estilo epistolar. 
De estas cuatro obras es esta gramática la que resulta de especial interés por ser un instrumento necesario para contribuir al desarrollo y conocimiento del latín en Barcelona.

Y sobre todo por la valiosa información que ofrece su colofón, donde se dice que esta "gramática, que había arribado a la costa de Barcelona procedente del botín de unos piratas, cuando Juan Peyro, secretario del rey de Aragón, que, aunque hispano sabía latín y se había educado en el Lacio, y otros hombres instruidos la leyeron, muy impresionados, la mandaron imprimir por Johannes de Salsburgo y Pablo de Constancia, alemanes, que entonces estaban allí. Y pusieron todo su empeño en procurar en beneficio de la patria (a quien todo se debe) esto: que de tosca se hiciera culta, y en lugar de la barbarie se adornara con latinidad".

Este colofón revela entre otras cosas, la existencia en Barcelona de un grupo de intelectuales cultos, instruidos, alguno de los cuales era miembros de la cancillería real, con conocimientos de latín y formado en Italia. El único que aparece mencionado con nombre y apellidos es Joan Peyró, secretario del rey de Aragón, que, aunque español, sabía latín y se había educado en el Lacio, del que sabemos llegó a ser lugarteniente del protonotario, cargo que, en tiempo de Alfonso el Magnánimo y de su hijo Ferrante, implicaba la residencia en Barcelona con ocasionales viajes a Nápoles (Sevillano Colom, 1965: 199). Rubio i Balaguer (1993: 111-112) señala la existencia de dos personas con el mismo nombre en el siglo XV, ambos al parecer funcionarios de la cancillería, lugartenientes del protonotario del rey de Aragón. Según Griffin Peyró fue alumno del humanista Arnau Fenolleda, secretario y protonotario de Alfonso V el Magnánimo (Sevillano Colom, 1965: 189192).

Según el colofón, la publicación de la gramática de Perotti en Barcelona, fue solo fruto de la casualidad: se encontró la obra en la playa procedente de un botín pirata.

Sin querer restar veracidad al texto es posible que Joan Peyró, dada su formación y sus contactos con Nápoles, tuviera conocimiento de la gramática bien por un ejemplar de la primera edición (Roma: Conrad Sweynheym y Arnold Pannartz, 19 de marzo 1473) (ISTC: ip00300000), bien por la segunda edición (Roma: Giovanni Filippo de Lignamine, 10 mayo 1474) (ISTC: ip00301000). En cualquier caso, fue un ejemplar de la edición romana de Lignamine el que sirvió de modelo para la edición de los Rudimenta Grammatices barcelonesa ya que conserva su prefacio, muy habitual en sus trabajos pero que ha desaparecido en otras ediciones, y su hechura.

Es también posible que Peyró tuviera ocasión de conocer al mismo Lignamine, activo en Nápoles hasta 1472 y muy próximo al rey Ferrante ${ }^{5}$ y al círculo de humanistas de la corte napolitana, entre los que estaba su maestro Antonio Beccadelli, el Panormita. No hay que olvidar que es obra suya la biografía, Inclyti

\footnotetext{
Lignamine era natural de Mesina. Se formó en Nápoles en la corte de Alfonso V. Al parece fue educado en el mismo círculo que Ferrante con humanistas como el Panormita. Su proximidad con el futuro rey le permitió escribir después su biografía, Inclyti Ferdinandi regis vita et laudes. En torno a 1472 se trasladó a Roma donde estableció su taller de impresión. El 27 de noviembre de 1473 salió del taller de Lignamine la segunda edición de las Epistolae Magni Turci de Laudivio Zacchia, dos meses después de la primera edición napolitana, y poco después, el 10 de mayo de 1474 los $R G$. Una biografía más completa en Alaimo (1988).
} 
Ferdinandi Regi vita et laudes, además de su editor e impresor (Roma: Giovanni Filippo de Lignamine, 1472) (ISTC: il00214500).

Y que igualmente tuviera noticia de las Epistolae Magni Turci de Laudivio Zacchia, ya que la obra había despertado interés tanto por el tema, que hacía referencia a una situación política real en el Mediterráneo ${ }^{6}$, como por constituir un modelo práctico del ars dictaminis, con cartas breves y de sintaxis sencilla, fáciles de imitar. En este contexto la edición de las Epistolae vendría a completar el elenco de obras provechosas encaminadas a revertir la situación cultural barcelonesa de incultura a la que se alude en el colofón de los Rudimenta.

Como en el caso de los Rudimenta la edición barcelonesa de las Epistolae sigue la hechura de la edición romana de Lignamine de 1473, que prescinde del poema De Hermaphrodito del Panormita, y utiliza una tipografía romana (R125), lo que hace pensar que fue un ejemplar de esta obra la que se tomó como modelo para la edición.

Tampoco se puede descartar la posibilidad de que Joan Peyró conociera al propio Zacchia, presente en Nápoles desde 1471, por su doble condición de hombre de letras y de armas. Igual que Niccolò Perotti, Laudivio se había formado en Ferrara con Guarino de Verona $^{7}$ y estaba relacionado con dos de los humanistas que formaron parte del círculo de intelectuales de la corte napolitana: conocía a Antonio Beccadelli, el Panormita (1394-1471), a quien Laudivio quiso rendir homenaje incluyendo en la primera edición de sus Epistolae un poema desconocido de su colección de poemas De Hermaphrodito, y a Giovanni Pontano (1426-1503) ${ }^{8}$, quien le animó a trasladarse a Nápoles en torno a 1471. Zacchia por otra parte, era caballero, eques Hierosolimitanus ${ }^{9}$, de San Juan de Jerusalén, orden militar con estrechos lazos con Cataluña y Aragón durante el siglo XV (Durán i Duelt, 2009: 97112).

El círculo de relaciones tal vez se pueda cerrar con Francesc Beltrán, a quien Laudivio Zacchia dirige el prefacio de su Vita Beati Hieronymi (Nápoles: Sixtus Riessinger, 1473) y de las Epistolae Magni Turci. Apenas hay información sobre la

6 El único príncipe occidental al que menciona Zacchia por su nombre es precisamente al rey Ferrante de Nápoles. Además, aunque las cartas son literarias, no se debe olvidar que el peligro turco era real y que él era un caballero de la orden sanjuanista, muy afectada por los avances del turco. Poco antes del asedio de Rodas en 1480 escribió una carta al cardenal de Pavía Jacobo Ammaniti Piccolomini (1422-1479), exhortando vivamente a la defensa de la ciudad y haciendo referencia a la caída de Caffa (turca desde 1475). Por tanto, es posible que no fuera solo un ejercicio retórico sino también un deseo real de formar una liga contra el turco, algo que ya había intentado el papa Pío II.

7 Laudivio Zacchia frecuentó la corte de Borso de Este, duque de Ferrara, a quien dedicó una tragedia en latín, De captivitate ducis Jacobi tragoedia (1465), que cuenta lo que sucedió a Jacobo Piccinino, famoso condotiero, hecho prisionero en 1464 y muerto en 1465 por orden de Ferrante de Nápoles. El duque de Ferrara, Borso de Este, figura como uno de los personajes. Posteriormente Laudivio se trasladó a Roma con el papa Nicolás V con quien al parecer tuvo mucho trato y familiaridad (intimus fuit et familiaritate et cubiculo). Se trasladó a Nápoles no antes de 1471.

8 Se conserva en la Biblioteca Vaticana una Epistula commendataria Ioviano Pontano Oboedientiae Charonisque (mss. Urb.lat.1401) donde Laudivio alaba tanto el De oboedientia como los Dialogi qui Charon et Antonius inscribuntur. Empieza así: Laudivius eques Hierosolimitanus s. Pontano Suo commendatio Oboedientae Charonisque. La primera edición que se conserva del De oboedientia de Pontano (Nápoles, 1490) no incluye esta epístola.

9 Título con el que se denomina en sus obras impresas, las Epistolae y la Vita Beati Hieronimi, si bien no se sabe en qué momento ingresó en la orden sanjuanista. 
persona que se oculta tras este nombre, bastante común en la Cataluña de la época (Iglesias i Fonseca 2008) ${ }^{10}$ que pudiera estar también vinculado a la Baronía de Gélida (Catalá i Roca, 1999: 381-390). A falta de información, las noticias más certeras de que disponemos proceden de dichos prefacios. Según estos Francesc Beltrán era natural de Barcelona y "comes”, compañero o conde, según se quiera interpretar ${ }^{11}$. De los textos, dos prólogos cargados de referencias a los autores clásicos tanto latinos como griegos, se desprende que se trataba de un hombre con formación y cultura, con quien el autor tiene estrecha relación intelectual. Según señala Zacchia en el prefacio de las Epistolae, fue Francesc Beltrán quien le animó a escribir y a publicar tanto la vida de San Jerónimo como el epistolario: "Mi querido Francesc, he aquí las cartas que he dado a luz hace poco. No tengo a nadie a quien enviar mejor que a ti... como las cartas son fruto de mi ocio, no he puesto al escribirlas todo el cuidado que requerían, y si las he dado a luz es por creer que os daría gusto al daros razón de mi retiro y mis estudios”.

Ambos compartían el interés por los manuscritos, los libros antiguos y las librerías, de tal manera que si Zacchia figura como posesor de varios códices griegos que al parecer adquirió en la isla de Chipre en torno a 1469 (Browning; Constantinides, 1993: 20, 209, 211), el nombre de Francesc Beltrán figura vinculado a un manuscrito del De officiis de Cicerón que había pertenecido previamente al notario Bernat d'Esplugues, cuya biblioteca despertó el interés del rey Alfonso el Magnánimo (Iglesias i Fonseca, 2000: 85-119).

Todo esto permite situar a Francesc Beltrán, natural de Barcelona, en ese grupo de ciudadanos anónimo, integrado por hombres cultos, ilustrados de Barcelona, miembros de la cancillería real, cuyo papel de mecenazgo intelectual no se limitó a la edición de la gramática de Perotti, sino que alentó también la edición del resto de las obras, entre las que debemos situar las Epistolae Magni Turci, que salieron del taller barcelonés de Hurus y Johannes de Salzburgo en esa misma época.

Aunque el nombre de Francesc Beltrán solo figura en los prefacios de las obras de Laudivio Zacchia, el nombre de Joan Peyro aparece vinculado años más tarde a la edición del Libellus inscriptus Barcinona, una descripción de la ciudad, obra de Jeronimus Paulus, impreso por Pere Miquel en Barcelona en 1491. La obra está dedicada a Paulus Pompilius, conocido por ser autor de una biografía de Séneca el joven y un comentario a las vidas de Augusto y Tiberio (Lee, 1978: 185).

Tanto el análisis tipográfico como el contexto cultural y social permiten justificar la edición de las Epistolae Magni Turci en Barcelona, dentro del conjunto de obras con vocación didáctica que salen del taller barcelonés de Hurus en 1475.

10 En la documentación manejada por Iglesias aparece varias veces el nombre de Francesc Beltran en diferentes inventarios de 1438: un Francesc Beltran, “canonige de la Seu”; en otro de 1457 aparecen un "sabater" y otro "cavaller".

11 En las ediciones de la Vita, Francesc es mencionado como barcelonés “Ad Francinum Beltrandum Barchinonensem”. En las primeras ediciones de las Epistolae solo figura el nombre. En cambio, en la edición de Padua de 1475 de la misma obra se añade su condición de “comes”: "Laudivii equitis Hierosolimitani ad Francinum Beltrandum comitem in epistolas magni turci”. Esto se repite en la edición de Treviso de 1475, en la de Roma de 1483, en las de Leipzig de 1488, 1498 y 1500 y en la de Würzburg de 1495. 
Será en cambio el contexto político el que justificará una nueva edición de la obra en Zaragoza entre 1479-1481, la segunda en España y la segunda de Hurus, coincidiendo con el asedio en 1480 a Rodas, principal destino comercial de la península durante el siglo XV y con gran presencia hispánica (Duran i Duelt, 2009: 106). No hay que olvidar que es en esa misma época cuando salen del taller de Hurus obras como la Obsidionis Rhodiae descriptio de Caorsin (1481) y las Bula de indulgencias en favor de la Santa Cruzada para la defensa de Rodas en 1479 y en 1481 en castellano y latín.

\section{Conclusiones}

El estudio de las fuentes conservadas permite identificar una nueva edición de las Epistolae Magni Turci en España desconocida hasta el momento.

El descubrimiento de esta nueva edición lleva a una reinterpretación de los datos existentes y de la datación de la edición conocida hasta la fecha. El análisis tipográfico y el contexto cultural la sitúan definitivamente en Barcelona en el taller que Hurus tiene en 1475 coincidiendo con la impresión de obras en latín con un claro enfoque didáctico.

Esta nueva datación la sitúa entre las cinco primeras ediciones que se hicieron de la obra en Europa. De esta edición se conservan tres ejemplares: uno en la John Rylands Library de Manchester y dos ejemplares en la Biblioteca Nacional de España.

La readscripción de la primera edición de la obra a Barcelona pone de manifiesto una actividad cultural importante en la ciudad por aquellas fechas, muy vinculada a los grupos culturales napolitanos. Confirma también la existencia de un grupo de personas cultas, letradas, entre las que cabe incluir tanto a Joan Peyró como a Francesc Beltrán, que actuarían como mecenas o impulsores de diferentes ediciones, entre las cuales se incluyen las Epistolae que tiene su modelo en la edición romana de Lignamine, autor, editor e impresor, muy relacionado con Ferrante de Nápoles.

Por su parte la nueva edición realizada en Zaragoza, sale del taller de Hurus por razones más bien políticas, en torno a 1479-1481, coincidiendo con el asedio a Rodas. De esta edición en caracteres góticos se conservan dos ejemplares, uno en la Biblioteca de Cataluña y otro en la Biblioteca Vaticana (Véase Figura 14).

\begin{tabular}{|l|l|}
\hline Primera edición: Barcelona, 1475 & Segunda edición: Zaragoza, c. 1479-1481 \\
\hline $\begin{array}{l}\text { Impresor: Paulo Hurus y Johannes } \\
\text { de Salsburgo }\end{array}$ & Impresor: Paulo Hurus y Johannes Planck \\
\hline $\begin{array}{l}\text { Ejemplares: Madrid BNE I/2588. } \\
\text { Madrid. BNE I/395. Manchester. John } \\
\text { Rylands Library JRL 18486. }\end{array}$ & $\begin{array}{l}\text { Ejemplares: Biblioteca de Catalunya 9-V-62. C. } \\
\text { del Vaticano BVat.(Stamp.Chig.IV.1251(3). }\end{array}$ \\
\hline
\end{tabular}

Figura 14: Ediciones de las Epistolae realizadas por Hurus y ejemplares localizados. 


\section{Descripción bibliográfica de las dos ediciones de Paulo Hurus:}

\section{Primera Edición}

Zacchia, Laudivius.

Epistolae Magni Turci. [Barcelona: Paulo Hurus y Johannes de Salsburgo, 1475].- $4^{0}$. - - a - $b^{8} c^{6} d^{8}$. - 30 h. - Letra romana (R110-QG). - 21-23 líneas.

Signaturas: con números en romanos y arábigos en el tercer pliego (a3, b3, c3, d3). Delante de cada letra y de cada número y tras el número, un punto (.) $\begin{array}{lllllll}\text { Sin foliación. } & \text { En blanco h. } & 29 \mathrm{v} & \mathrm{y} & \mathrm{h} . & 30\end{array}$ Espacios en blanco para iniciales sin letras guía.

Letras mayúsculas en todas las rúbricas, idéntica a la empleada en la edición napolitana de 1473, con puntos para separar palabras.

a1r, 1. 1: LAUDIVII equitis hieroslymitani ad // Francinū Beltrandū in epistolas magni // Turci praefatio. l. 4: [E]pistolas a me nup in lucem // editas: ad quē potius miterē // Frācine

d7r, l. 15-16: tior uictoria est quam nullo rei.p. detri// mento: patriā ab îcendio belli seruasse. 1. 17: FINIS.

\section{Referencias:}

Accurti (1930) 96; CCPB000110692-9; García Rojo, 1945: 1125; GW M25638; GW M25618; IBE 3457; ISTC im00059500; Martín Abad L-14; Pallarés 9; Vindel, 1946: IV, 6.

\section{Ejemplares:}

Madrid BNE I/2588. Madrid. BNE I/395. Manchester. John Rylands Library JRL 18486.

\section{Reproducciones:}

Biblioteca Digital Hispánica (Biblioteca Nacional de España) I/2588

\section{Segunda edición}

Zacchia, Laudivius Epistolae Magni Turci. [Zaragoza: Paulus Hurus y Johannes Planck, entre 1479-1481].—4 -_ — [24] h. —Letra gótica (104G: M17E). — 26-27 líneas.

Sin signaturas. Colación: [a-c]8: a1 falta, pero presumiblemente en blanco, c7v y c8 en blanco.

Sin foliación. En bl. h. 23v y h. 24.

Espacios en blanco para iniciales sin letras guía. 
a2 r, 1. 1: Laudiuii equitis hjeroslimitani ad Frā // cinū Beltrandum in epistolas magni tur // ci prefatio. l. 4: [E]pistolas a me nup in lucem edi///tas. ad quē potius mittere3 Fran // cine.

c7r, l. 3-5: victoria est $q$ // nullo rei.p. detrimento: patriam ab incē / //dio belli seruasse. 1. 6: Finis Epistolarum Laudiuii.

Referencias (las mismas que para la edición anterior):

CCPB000110692-9; GW M25618; GW M25638; IBE 3457; ISTC im00059500; Martín Abad L-14; Pallarés 9; Vindel, IV, 6 [Hurus y Planck hacia 1479-1484]; Vindel(A) IV 23: 6.

\section{Ejemplares:}

Barcelona. Biblioteca de Catalunya 9-V-62. Ciudad del Vaticano BVat. (Stamp.Chig.IV.1251(3).

\section{Reproducciones:}

Barcelona. Biblioteca de Cataluña Memòria Digital de Catalunya 9-V-62.

\section{Repertorios bibliográficos:}

BMC: British Library (1971). Catalogue of books printed in the XVth century now in the British Museum. Part X: Spain and Portugal. London: The Trustees of the British Museum.

CCPB=Catálogo Colectivo del Patrimonio Bibliográfico Español (1985-). [recurso on line] (última versión 2019) http://catalogos.mecd.es/CCPB/cgiccpb/abnetopac [Consulta: 20/11/2019].

García Rojo, D.; Ortiz de Montalván, G. (1945). Catálogo de incunables de la Biblioteca Nacional. Madrid: Tip. de Blass.

Griffin, N. (1988). Spanish Incunabula in the John Rylands University Library of Manchester. Bulletin of the John Rylands University Library, 70 (2), 3-143.

GW= Staatsbibliothek zu Berlin (1968-). Gesamtkatalog der Wiegendrucke. Bd. I [etc.] Stuttgart, etc. (Vols. 1-7 reproduced with additions and corrections from the original edition (Leipzig, etc., 1925-38) [recurso on line] /www.gesamtkatalogderwiegendrucke.de [Consulta: 1/12/2019].

IBE: Biblioteca Nacional de España (1989-1990). Catálogo general de incunables en bibliotecas españolas. Coordinado y dirigido por Francisco García Craviotto. Madrid: Ministerio de Cultura. Dirección General del Libro y Bibliotecas, 2 vols.

ISTC: Incunabula Short Title Catalogue: The international data base of 15thcentury European printing [recurso on line] (última versión 2019) https://data.cerl.org/istc/im00063600?style=_default [Consulta: 20/11/2019].

$T W=$ Typenrepertorium der Wiegendrucke. Base de datos de impresores y tipos de imprenta incunable de la Staatsbibliothek - Preußischer Kulturbesitz (Berlín). [recurso on line] https://tw.staatsbibliothek-berlin.de/html/index.xql [Consulta: $1 / 12 / 2019]$

Vindel, F. (1945-1951). El arte tipográfico en España durante el siglo XV. Madrid: Dirección General de Relaciones Culturales, 9 vols. 
Vindel, F. (1945). El arte tipográfico en España durante el siglo XV. T. I. Cataluña, Madrid: Dirección General de Relaciones Culturales.

Vindel, F. (1946). El arte tipográfico en España durante el siglo XV. T. IV. Zaragoza. Madrid: Dirección General de Relaciones Culturales.

\section{Referencias bibliográficas}

Alaimo, C. (1988). Giovanni Filippo da Lignamine, Dizionario Biografico degli Italiani, 36.www.treccani.it/enciclopedia/de-lignamine-giovanni-filippo_\%28Dizionario-Biogra fico\%29/ [Consulta: 20/11/ 2019].

Alessandro, P. D'. (2015). Niccolò Perotti, Dizionario Biografico degli Italiani, 82. www.treccani.it/enciclopedia/niccolo-perotti [Consulta: 20/11/2019].

Babinger, F. (1960). Laudivius Zacchia, der Erdichter der Epistolae Magni Turci. Bayerische Akademie der Wissenschaften, Phil.-hist. Klasse, Sitzungsberichte, 13, 1-43.

Bentley, R. (1697). Dissertation upon the Epistles of Phalaris, Themistocles, Socrates, Euripides and others, and the Fables of Aesop. London: Printed by J. Leake.

Browning, R.; Constantinides, C. N. (1993). Dated Greek Manuscripts from Cyprus to the Year 1570. (Dumbarton Oaks Studies $\mathrm{n}^{\circ}$ 30). Washington D.C.-Nicosia: Dumbarton Oaks Research Library and Collection.

Buffardi, G.; D’Agostino, G., eds. Lit. (2000). La Corona d'Aragona ai tempi di Alfonso il Magnanimo: I modelli politico-istituzionali, la circolazione degli uomini, delle idee, delle merci; Gli influssi sulla società e sul costume. Congreso de Historia de la Corona de Aragón (16. 1997. Nápoles, etc.). Napoli: Ed. Paparo.

Catalá i Roca, P. (1999). Contribució a l'estudi dels Beltran (s. XV) Familia senyorial de Gelida. Acta historica et archaeologica mediaevalia, 20-21, 381-390.

Duran i Duelt, D. (2009). Presencia hispánica en Rodas. A propósito del Albergue de la lengua de España. MRAMEGH, 19, 97-112.

Iglesias i Fonseca, J. A. (2008). Llibres i lectors a la Barcelona del segle XV. Les biblioteques de clerges, juristes, metges i altres ciutadans a través de la documentació notarial (anys 1396-1475). Tesis doctoral. Bellaterra: Universidad Autònoma de Barcelona. www.tdx.cat/handle/10803/5549\#page=1 [Consulta: 1/12/ 2019].

Iglesias i Fonseca, J. A. (2000). Els clàssics a la biblioteca de Bernat d’Esplugues (†1433), notari i escrivà del Consell de la Ciutat. Faventia, 22 (2), 85-119.

Iglesias i Fonseca, J. A. (2001). El ms. S.II.26 de El Escorial (o la desaparición de textos de Cicerón de un códice propiedad del notario Bernat d'Esplugues, m. 1433). Convenit Selecta, 7: Cicero And The Middle Ages, 91-98. www.hottopos.com/convenit7/ iglesias.htm [Consulta: 1/12/2019]

Lee, E. (1978). Sixtus IV and Men of Letters. Roma: Ed. Di Storia e Letteratura.

Meserve, M. (2008). Empires of Islam in Renaissance Historical Thought. Cambridge (Massachuset) - London: Harvard University Press.

Morales i Roca, F. J. (1988). Caballeros de la Espuela Dorada del Principado de Cataluña. Madrid: Ediciones Hidalguía.

Neri, A. (1875). Lettera di Laudivio da Vezzano sulla caduta di Caffa. Nota bibliografica delle sue opere e correzione intorno al suo cognome. Giornale Linguistico di archeologia storie e belle arti, 2, 137-153.

Pallarés, M. Á. (2008). La imprenta de los incunables de Zaragoza y el comercio del libro en Zaragoza a finales del siglo XV. Zaragoza: Institución Fernando El Católico. 
Rubio i Balaguer, J. (1993). Llibreters i impressors a la Corona d'Arago. Barcelona: Publicacions de l'Abadia de Montserrat.

Sevillano Colom, F. (1965). Cancillerías de Fernando I y de Alfonso V. Anuario de historia del derecho español, 35, 169-216.

Tentori, P. (1962). Arnaldo de Bruxelles, en: Dizionario Biografico degli italiani, 4 [recurso on line] hwww.treccani.it/enciclopedia/arnaldo-da-bruxelles_(DizionarioBiografico)/ [Consulta: 20/11/ 2019]. 